\title{
oints
}

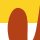

\section{Treatment of juvenile osteochondritis dissecans of the talus: current concepts review}

\author{
FRANCESCA VANNINI, MARCO CAVALLO, MATTEO BALDASSARRI, FRANCESCO CASTAGNINI, \\ ALESSANDRA OLIVIERI, ENRICO FERRANTI, ROBERTO BUDA, SANDRO GIANNINI
}

$1^{\text {st }}$ Clinic of Orthopaedics and Traumatology, Rizzoli Orthopaedic Institute, Bologna, Italy

\begin{abstract}
Juvenile osteochondritis dissecans of the talus (JODT) affects the subchondral bone primarily and, in a skeletally immature population, articular cartilage secondarily. It probably consists of aseptic bone necrosis whose spontaneous healing is impaired by microtraumas, resulting in an osteochondral injury and, in some cases, in osteoarthritis. In many cases the clinical presentation is asymptomatic. Mild chronic pain is frequent, sometimes accompanied by swelling, stiffness or locking. Few data are currently available on this topic and, moreover, most existing data were obtained from mixed groups and populations; it is therefore difficult to outline a scheme for the treatment of JODT. However, the most suitable treatment in the first stages of the disease is conservative. The presence of a loose body is an indication for surgical fixation, drilling or regenerative procedures, depending on the presence/extent of subchondral bone sclerosis and the surgeon's experience. Drilling has been shown to promote the healing of lesions with minimal surgical trauma. Microfractures, since they induce fibrocartilage repair, are to be considered only for small injuries. Mosaicplasty and osteochondral autograft transplantation may cause donor site morbidity and are techniques little reported in JODT. Regenerative techniques and fresh allografts give good results in osteochondral lesions, but further studies are required to describe the results that can be obtained in JODT alone.
\end{abstract}

Key Words: ankle, juvenile, osteochondritis dissecans, microfractures, regenerative techniques.

\footnotetext{
Corresponding Author:

Francesco Castagnini, MD

$1^{\text {st }}$ Clinic of Orthopaedics and Traumatology, Rizzoli Orthopaedic Institute

Via Giulio Cesare Pupilli 1, 40136 Bologna, Italy

E-mail: francescocastagnini@hotmail.it
}

\section{Introduction}

Osteochondritis dissecans (OCD) of the talus is a disease affecting the subchondral bone and secondarily the articular cartilage. The ankle is the third most frequently affected site, after the knee and elbow, and it accounts for $4 \%$ of all OCD cases $(1,2)$. OCD of the talus usually occurs in patients aged between 10 and 40 years, and it peaks in the second decade of life. When this disease occurs before physeal closure, it is called juvenile osteochondritis dissecans of the talus (JODT) (2).

Clinically, patients with JODT usually complain of mild chronic pain, occasional stiffness or swelling and, rarely, locking or catching due to the presence of a loose body (3, 4). MRI is the gold standard for JODT diagnosis $(2,5)$ (Fig. 1). Although MRI is highly accurate when compared to arthroscopy (sensitivity: 92\%, specificity: 55\%), it may nevertheless give false-positive results when used to detect JODT. In particular, MRI may give false-positive results when it is used to assess the instability of the osteochondral fragments (2). In these cases, CT may provide a better definition of the state of the subchondral bone (2).

JODT is regarded as aseptic bone necrosis caused by repetitive microtraumas in a predisposed individual $(6,7)$. In the juvenile population, spontaneous healing may frequently occur. When this process does not take place, the inadequate integration of the bone is due to the interposition of sclerotic tissue which provides a barrier to the blood supply and, consequently, to the healing process driven by subchondral stem cells. Initially, the osteochondral fragment is anchored by the continuous surface of hyaline cartilage and the sclerotic tissue. However, as a result of subsequent cartilage degeneration, the fragment becomes a loose body in the ankle joint (8). These lesions may even evolve into a clinically evident ankle deformity and instability, mostly in cases of extensive necrosis $(9,10)$.

It is crucial to establish the natural history of JODT in order to plan the correct treatment: if, in the presence of 
viable cartilage, the fragment is not displaced, then the rate of healing is very high and surgery is not required. Vascularization is severely restricted when fragments are displaced, in which case surgery may be the only way to heal the lesion. Joint stability and a small lesion size seem to be other important positive prognostic features $(5,11)$. This review looks at the current options for treating JODT, focusing on the correct surgical indications, the advantages of the available techniques, and the future perspectives for the treatment of this disease.

\section{Literature review}

A literature search of the PubMed database was conducted, including all papers, published in English, dealing with conservative and surgical treatments of OCD. The following search terms were used: "osteochondritis dissecans", "transchondral fractures", combined with the terms "ankle", "talus" and "juvenile", "adolescent", "children". Little evidence on this topic is available to date, as many articles do not deal strictly with JODT, instead mixing adult and juvenile populations, OCD and osteochondral lesions. The articles were also reviewed for information on a possible therapeutic scheme.

\section{Preliminary considerations}

JODT is a benign disease with a relative high rate of spontaneous healing and a low rate of joint degeneration (8). The current treatments are based on the classification proposed by Berndt-Hardy for osteochondral lesions of the talus and then adapted to JODT; this approach is based on radiographic findings (9). In the first stage, a small area of compression of subchondral bone can be detected. When an osteochondral fragment is partially detached, the disease is classified as second stage. The third stage is characterized by a completely isolated, but not displaced, fragment. The fourth stage includes loose bodies.

The treatment of JODT should aim to achieve preservation of articular cartilage and healing of the damage as soon as possible: growth plate closure turns JODT into adult OCD, with a consequent poorer prognosis (9).

\section{Conservative treatment}

Consistent evidence exists regarding the non-surgical treatment of stable and small fragments in patients with open growth plates (4, 8-11). In JODT, irrespective of the disease stage, the site of the lesion should always be evaluated carefully to weigh up the possibility of a conservative treatment. Indeed, some surgical treatments may involve osteotomy and this, in growing children, can lead to limb length discrepancy or deviation (9). On the other hand, conservative treatments may take a very long time to achieve complete healing (11).

Higuera et al. (9) treated $68 \%$ of patients with JODT conservatively. Most of them were classified as stage III. Cast and no weight bearing were recommended for at least six weeks, followed by three months of active movements with no weight bearing. One case required surgery for poor outcomes. Overall, the results (clinical and radiological parameters) were rated as good or excellent. The outcomes after conservative treatment were also satisfying in the studies by Perumal et al. (3) and Letts et al. (4).

\section{Surgical treatment}

Surgery is recommended when conservative outcome is not satisfactory, the fragment is displaced or large, and sclerosis prevents the lesion from healing spontaneously $(3,4,9,12)$. In the most import work on JODT, by Higuera et al. (9), the surgical procedures performed were bone marrow stimulation and/or fixation. The rehabilitation included a short period of immobilisation, followed by active movements with no weight bearing. Considering all the cases, treated conservatively and surgically, $94 \%$ of the patients achieved excellent or good clinical results, with a remarkable reduction of pain, even during exercise, and a full, or minimally restricted, range of movement, and $68.5 \%$ of radiological results were classified as excellent or good. Among bone marrow stimulation techniques, drilling should be mentioned as it

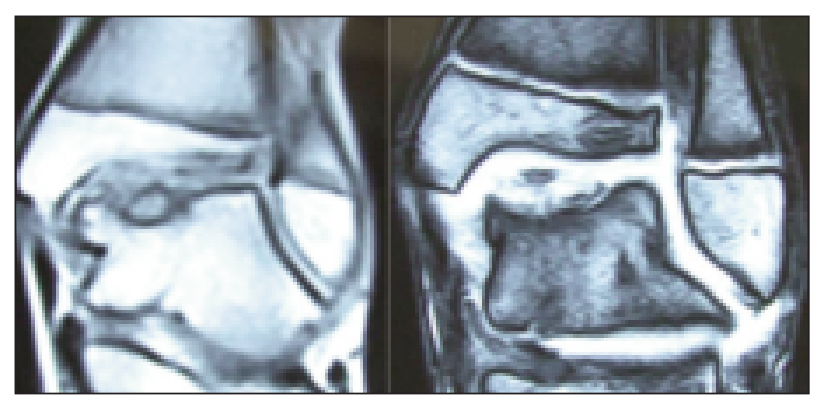

Fig. 1. Coronal views of pre-operative MRI in an 11-year-old female with Berndt-Hardy stage III disease. The case was treated with bone marrow-derived cell transplantation, a regenerative technique. 


\section{oints}

F. Vannini et al.

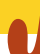

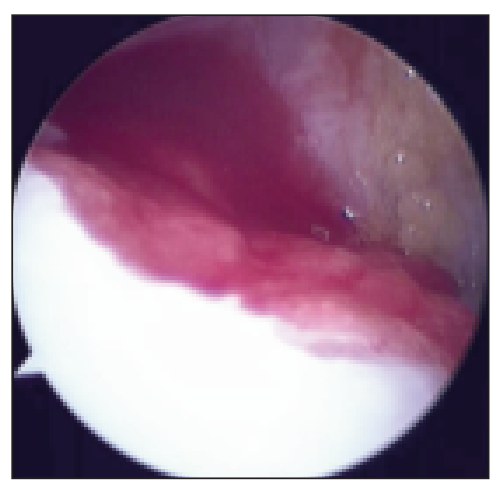

Fig. 2. Bone marrowderived cell transplantation performed for a JODT in a 12-year-old male, using a collagen membrane and autologous mononucleated stem cells.

offers several advantages in non-healing stable lesions (13). The surgical procedure requires a Kirschner wire to create channels in the subchondral bone, through which bone marrow cells can penetrate the articular space, stimulating the healing of the lesion. The drilling approaches may be anterograde or retrograde. The anterograde approach runs the pin into the lesion through the medial malleolus, and thus poses a risk to the cartilage overlying the lesion. The retrograde approach is friendlier to cartilage and growth plates. The wire penetrates the talar dome from the head of the talus, sparing the ankle surface. The disadvantages of this technique are technical difficulties, possible displacement of the fragment, and possible soft tissue injuries. Healing was reported in $90 \%$ of the cases after drilling, regardless of the approach (13).

Excessive sclerosis of the subchondral bone may impair healing even after drilling. Other techniques may be used successfully: fragment excision, microfractures, mosaicplasty, osteochondral autograft transplantation (OATS), autologous chondrocyte implantation (ACI), or bone marrow-derived cell transplantation (BMDCT) $(10,12)$. The arthroscopic approach should be preferred, taking care to ensure that in JODT the joint (due to its small dimensions) and the growth plates are protected against harmful stresses; for this reason, dedicated instrumentation is required. Fragment excision is questioned, even though it was successfully used in a JODT case series (3, $4,9)$. In multiple works dealing with mixed osteocartilagineous lesions of knee and ankle, this procedure was reported to provide acceptable short-term results, with a risk of joint degeneration at long term follow-up. In some cases, a detached osteochondral fragment may be viable and its fixation may restore joint surfaces and avoid ankle osteoarthritis (14).

The microfracture procedure may be a good option for small JODT lesions, but no studies are currently available. When applied to OCD of the knee, $41 \%$ of failures after microfractures were reported (15). Good short-term results have been widely reported (16). At mid-term follow-up, deteriorations in clinical and MRI outcomes were evident, particularly if compared to the outcomes of a regenerative technique like osteochondral autologous transplantation (15). The main concern with microfractures relates to the healing of the lesion through fibrocartilage, which is a poor quality tissue with modest durability. Thus, this procedure should be used only for small lesions, even though the simplicity of the technique makes this treatment attractive $(15,16)$. JODT may be effectively treated using regenerative techniques, in order to restore a hyaline-like chondral layer, which is fundamental to avoid osteoarthritis at long-term follow-ups. Nevertheless, there are only few reports of the use of these techniques in knee joints.

Autologous osteochondral transplantation, such as mosaicplasty or OATS, has been used in OCD of the knee and osteochondral lesions of the talus, with interesting results $(15,17)$. It is a regenerative technique involving restoration of hyaline cartilage with fibrocartilage filling of the gaps between the plugs. Due to the problems of the limited donor area (non-weight-bearing area of the ankle) and donor site morbidity, only small lesions $\left(1-5 \mathrm{~cm}^{2}\right)$ should be treated using this technique. Other limits of this technique may include the need for a stable talus shoulder, which is crucial for the fixation of the plugs. Moreover, this procedure is quite technically demanding, requires dedicated instrumentation, and is usually performed by open approach $(15,17)$.

ACI may be a valuable option in JODT. It was used in juvenile OCD of the knee, with successful clinical results, regardless of the dimensions of the lesions (18). The arthroscopic findings at one- year follow-up demonstrated fibrocartilage repair in half of the cases, and a mixture of hyaline and fibrous cartilage in the other half. Ankle ACI instead has been proposed only in ankle osteochondral lesions, giving encouraging results (19). BMDCT with a one-step arthroscopic technique (20, 21) was successfully proposed for the treatment of OCD of the knee and osteochondral lesions of both the knee and the ankle (Fig. 2). Thanks to the multipotential ability of bone marrow nucleated cells, in association with platelet-rich fibrin (PRF), the osteochondral layer may regenerate.

Cells are collected from spongy bone of the posterior iliac crest and concentrated directly in the operating room, isolating the nucleated, multipotent cells. At the same time, an ankle arthroscopy is performed, debriding 
necrotic tissue and eliminating impingement. Then, $2 \mathrm{ml}$ of the concentrated cells are loaded on a scaffold (e.g. hyaluronan or collagen membrane), already prepared, in size and shape, to match the dimensions of the lesion. Dedicated instrumentation is used to position the biomaterial on the lesion. Then, PRF is sprayed on the lesion in order to secure the implant and promote the growth and differentiation of the cells. The rehabilitation programme requires a non-weight-bearing period of 4-6 weeks, and active and passive movements. After 6 months, light running is permitted and 10 months after the surgery high-impact sports are allowed. Good clinical and radiological outcomes have been achieved in JODT, too, but the results are still unpublished.

In the case of extremely large lesions with incomplete disruption of the talar anatomy, a partial fresh allograft is possibly the most reasonable solution. Lyon et al. (22) described the use of this technique in the treatment of juvenile OCD of the knee (not all the patients were skeletally immature). The clinical and radiological results were promising. No data exist for this technique in JODT, but a paper describing this technique in ankle osteochondral lesions reported satisfactory results (23).

\section{Conclusions}

JODT may be effectively treated with a conservative treatment in the first two Berndt-Hardy classification stages of the disease. Avoidance of weight bearing, with the ankle contained in a cast, or better, in a brace, is advisable. If the non-surgical treatment fails, drilling has given good results. Fragment excision should be avoided, whereas, in case of a viable detached loose body, fixation may achieve good results. Microfractures should be limited to small lesions. Regenerative techniques, in particular ACI and BMDCT, should be encouraged in order to restore a good, viable osteochondral layer, and avoid ankle joint degeneration.

\section{References}

1. Bruns JBP. Osteochondrosis dissecans. Arthroskopie. 1998; 11:166-176.

2. Heywood CS, Benke MT, Brindle K, et al. Correlation of magnetic resonance imaging to arthroscopic findings of stability in juvenile osteochondritis dissecans. Arthroscopy. 2011;27:194-199.

3. Perumal V, Wall E, Babekir N. Juvenile osteochondritis dissecans of the talus. J Pediatr Orthop. 2007;27:821-825.
4. Letts M, Davidson D, Ahmer A. Osteochondritis dissecans of the talus in children. J Pediatr Orthop. 2003;23:617-625.

5. Hughes JA, Cook JV, Churchill MA. Juvenile osteochondritis dissecans: a 5-year review of the natural history using clinical and MRI evaluation. Pediatr Radiol. 2003;33:410-417.

6. Steinhagen J, Niggemeyer O, Bruns J. Etiology and pathogenesis of osteochondrosis dissecans tali. Orthopade. 2001; 30:20-27.

7. Suckel A, Hoyer M, Raab C. Osteochondrosis dissecans and osteochondral lesions of the talus: clinical and biochemical aspects. Sportverletz Sportschäden. 2012;26:91-99.

8. Bauer M, Jonsson K, Lindén B. Osteochondritis dissecans of the ankle. A 20-year follow-up study. J Bone Joint Surg Br. 1987;69:93-96.

9. Higuera J, Laguna R, Peral M, et al. Osteochondritis dissecans of the talus during childhood and adolescence. J Pediatr Orthop. 1998;18:328-332.

10. Zwingmann J, Südkamp NP, Schmal H, et al. Surgical treatment of osteochondritis dissecans of the talus: a systematic review. Arch Orthop Trauma Surg. 2012;132:1241-1250.

11. Lam KY, Siow HM. Conservative treatment for juvenile osteochondritis dissecans of the talus. J Orthop Surg (Hong Kong). 2012;20:176-180.

12. Wall E, Von Stein D. Juvenile osteochondritis dissecans. Orthop Clin North Am. 2003;34:341-353.

13. Gunton MJ, Carey JL, Shaw CR, et al. Drilling juvenile osteochondritis dissecans: retro- or transarticular? Clin Orthop Relat Res. 2013;471:1144-1151.

14. Pascual-Garrido C, Tanoira I, Muscolo DL. Viability of loose body fragments in osteochondritis dissecans of the knee. A series of cases. Int Orthop. 2010;34:827-831.

15. Gudas R, Simonaityte R, Cekanauskas E, et al. A prospective, randomized clinical study of osteochondral autologous transplantation versus microfracture for the treatment of osteochondritis dissecans in the knee joint in children. J Pediatr Orthop. 2009;29:741-748.

16. Salzmann GM, Sah BR, Schmal H, et al. Microfracture for treatment of knee cartilage defects in children and adolescents. Pediatr Rep. 2012;4:e21.

17. Sasaki K, Matsumoto T, Matsushita T, et al. Osteochondral autograft transplantation for juvenile osteochondritis dissecans of the knee: a series of twelve cases. Int Orthop. 2012;36:2243-2248.

18. Krishnan SP, Skinner JA, Carrington RW, et al. Collagencovered autologous chondrocyte implantation for osteochondritis dissecans of the knee: two- to seven-year results. J Bone Joint Surg Br. 2006;88:203-205.

19. Giannini S, Buda R, Vannini F, et al. Arthroscopic autologous chondrocyte implantation in osteochondral lesions of the talus: surgical technique and results. Am J Sports Med. 2008;36:873-880.

20. Vannini F, Battaglia M, Buda R, et al. "One step" treatment of juvenile osteochondritis dissecans in the knee: clinical results and T2 mapping characterization. Orthop Clin North Am. 2012;43:237-44, vi.

21. Giannini S, Buda R, Cavallo M, et al. Cartilage repair evolution in post-traumatic osteochondral lesions of the talus: from open field autologous chondrocyte to bone-marrow-derived cells transplantation. Injury. 2010;41:1196-1203.

22. Lyon R, Nissen C, Liu XC, et al. Can fresh osteochondral allografts restore function in juveniles with osteochondritis dissecans of the knee? Clin Orthop Relat Res. 2013;471:11661173.

23. Hahn DB, Aanstoos ME, Wilkins RM. Osteochondral lesions of the talus treated with fresh talar allografts. Foot Ankle Int. 2010;31:277-282. 